TRANSACTIONS OF THE

AMERICAN MATHEMATICAL SOCIETY

Volume 329, Number 1, January 1992

\title{
MAXIMAL ENTROPY ODD ORBIT TYPES
}

\author{
WILLIAM GELLER AND JUAN TOLOSA
}

\begin{abstract}
A periodic orbit of a continuous map of an interval induces in a natural way a cyclic permutation, called its type. We consider a family of orbit types of period $n$ congruent to $1(\bmod 4)$ introduced recently by Misiurewicz and Nitecki. We prove that the Misiurewicz-Nitecki orbit types and their natural generalizations to the remaining odd periods $n$ have maximal entropy among all orbit types of period $n$, and even among all $n$-permutations.
\end{abstract}

\section{INTRODUCTION}

In this paper we study periodic orbit types with maximal entropy for interval maps.

A periodic orbit for a map $f$ of an interval determines a permutation, in fact a cycle, in a very natural way, via the order on the interval: if the orbit consists of $p_{1}<p_{2}<\cdots<p_{n}$, then we get the cycle $\theta$ on $n$ letters, where $f\left(p_{i}\right)=p_{\theta(i)}$. We call this cycle the type of the orbit. Two orbits of interval maps have the same type when there is an orientation-preserving homeomorphism of the intervals conjugating one orbit to the other. Orbit types have been studied by a number of authors in the last few years, for example [Ba, $\mathrm{Be}, \mathrm{BH}$, and $\mathrm{J}]$ in addition to [MN]. A good survey of topological dynamics on the interval is [N].

Topological entropy, defined by Adler, Konheim, and McAndrew [AKM], and characterized by Bowen [Bow], gives a measure of the complexity of a dynamical system. We can associate an entropy with an orbit type by taking the infimum of the topological entropies of all systems having an orbit of the given type. This quantity is positive for most orbit types, including all those whose period is not a power of two [BowF, BGMY]. We address here the question of which orbit types of a given period have the largest entropy.

The question of which orbit types have least entropy has been answered: work of Block and Coppel [BCop] shows that these are exactly the strongly simple orbit types defined in their paper.

Recently, Misiurewicz and Nitecki [MN] have obtained a beautiful asymptotic result on maximal entropy orbit types. They show that the exponential of the maximal entropy is asymptotically $2 n / \pi$. In proving this, they make use of an interesting new family of orbit types of period congruent to $1(\bmod 4)$, and

Received by the editors October 25, 1989.

1980 Mathematics Subject Classification (1985 Revision). Primary 58F08, 28D20; Secondary 26A18, 58F20, 54H 20.

Key words and phrases. Orbit type, entropy, cycle, Markov graph.

(C) 1992 American Mathematical Society $0002-9947 / 92 \$ 1.00+\$ .25$ per page 
they prove that these orbit types approach maximal entropy in the limit. They leave open the question of which orbit types attain maximal entropy.

We prove here that the orbit types of Misiurewicz and Nitecki, and their natural generalizations to the remaining odd periods, have maximal entropy. In fact, we prove more generally that these types have maximal entropy even among all permutations associated to unions of periodic orbits.

Briefly, the contents of the paper are as follows.

In $\S 2$ we give some basic definitions and results on orbit types and their entropies.

In $\S 3$ we define the generalized Misiurewicz-Nitecki orbit types, state the main result, and obtain some necessary results on maximal entropy types which are maximal in the natural partial preorder on permutations.

In $\S 4$ we define for each odd $n$ a cone in $\mathbf{R}^{n-1}$ that is used to estimate the entropy of various orbit types of period $n$, and prove the invariance of this cone under the square of the matrix corresponding to the Misiurewicz-Nitecki orbit type of period $n$.

In $\S 5$ we exploit this invariance together with other properties of the cone to conclude the proof of the main theorem.

We would like to thank Jim Propp for introducing us to orbit types and for his helpful comments, Jack Wagoner for his interest and encouragement, and Michał Misiurewicz and Zbigniew Nitecki for their generosity with early versions of their results. We would especially like to thank Ted Jones for his many valuable suggestions in the course of this work and for his expert computational help.

\section{Preliminaries}

We begin by fixing some notation and terminology. A map for us will be a continuous function $f$ from a compact interval $I$ into itself. The orbit of a point $x_{0} \in I$ is the sequence $\mathscr{O}=\mathscr{O}\left(x_{0}\right):=\left\{x_{i}\right\}_{i=0}^{\infty}$, where $x_{i}:=f^{i}\left(x_{0}\right)$ and $f^{0}$ is the identity map. Let $P_{n}$ be the set of permutations on $n$ letters, $P_{n}:=\{\theta:\{1, \ldots, n\} \rightarrow\{1, \ldots, n\} \mid \theta$ is bijective $\}$, and let $P:=\bigcup_{n \geq 1} P_{n}$. The set of cycles of length (or period) $n$ is $C_{n}:=\left\{\theta \in P_{n} \mid \theta^{k}(1) \neq 1\right.$ for $\left.0<k<n\right\}$. We let $C:=\bigcup_{n \geq 1} C_{n}$. The dual of a permutation $\theta \in P_{n}$ is the permutation $\bar{\theta} \in P_{n}, \bar{\theta}(i):=n+1-\theta(n+1-i)$, so that $\bar{\theta}$ is $\theta$ conjugated by a reversal of orientation. We will sometimes use cycle notation, so that $\theta \in C_{n}$ will be written as $\left(1 \theta(1) \theta^{2}(1) \cdots \theta^{n-1}(1)\right)$.

If an orbit $\mathscr{O}$ of a map $f$ is periodic, then we can write it as $\mathscr{O}=\left\{p_{1}, \ldots, p_{n}\right\}$ where $p_{1}<\cdots<p_{n}$. In this case the restriction of $f$ to $\mathscr{O}$ induces, in a very natural way, a cycle $\theta \in C_{n}$, which we call the orbit type of $\mathscr{O}$. Specifically, we define $\theta(i):=j$ if $f\left(p_{i}\right)=p_{j}$.

More generally, if $S=\left\{p_{1}, \ldots, p_{n}\right\}, p_{1}<\cdots<p_{n}$, and $f(S)=S$, we can define the type of the finite invariant set $S$ to be the permutation $\theta \in P_{n}$ given as before by $\theta(i):=j$ if $f\left(p_{i}\right)=p_{j}$.

The (topological) entropy of a map $f$ on a compact interval $I$ [AKM] is

$$
h(f):=\sup _{\mathscr{U}} \lim _{n \rightarrow \infty}(1 / n) \log M_{n}(\mathscr{U}),
$$

where for any open cover $\mathscr{U}$ of $I, M_{n}(\mathscr{U})$ is the minimum cardinality of a 
subcover of

$\mathscr{U}_{n}:=\mathscr{U} \vee f^{-1} \mathscr{U} \vee \cdots \vee f^{-(n-1)} \mathscr{U}$ and $\mathscr{U} \vee \mathscr{V}:=\{U \cap V: U \in \mathscr{U}, V \in \mathscr{V}\}$.

Equivalently [Bow],

$$
h(f)=\lim _{\varepsilon \rightarrow 0} \limsup _{n \rightarrow \infty}(1 / n) \log S_{n}(\varepsilon),
$$

where $S_{n}(\varepsilon)$ is the maximum cardinality of an $(n, \varepsilon)$ separated set. Here a set $E \subset I$ is $(n, \varepsilon)$ separated if for all distinct $x$ and $y$ in $E$, there is some $k$, $0 \leq k<n$, such that $\left|f^{k}(x)-f^{k}(y)\right|>\varepsilon$.

Definition 1. The entropy of an orbit type $\theta \in C$ is

$$
h(\theta):=\inf \{h(f): f \text { is a map with an orbit of type } \theta\} .
$$

More generally, if $\theta \in P$ we define $h(\theta):=\inf \{h(f): f$ has an invariant set of type $\theta\}$. It is easy to see that for all $\theta \in P, h(\bar{\theta})=h(\theta)$.

If $\theta$ is an orbit type, or more generally an invariant set type in $P_{n}$, we define the primitive function $f_{\theta}$ to be the piecewise linear interpolation of the graph of $\theta, f_{\theta}(x)=\{x\} \theta(\lceil x\rceil)+(1-\{x\}) \theta(\lfloor x\rfloor), 1 \leq x \leq n$. Here $\{x\}$ is the fractional part of $x$ and $\lceil x\rceil$ and $\lfloor x\rfloor$ represent the least integer as large as $x$ and the largest integer as small as $x$, respectively. $\theta \in P_{n}$ (or $f_{\theta}$ ) is maximodal if each $f_{\theta}(i)$ is a (local) extremum of $f_{\theta}$, for $1 \leq i \leq n$.

Proposition 1. For $\theta \in P, h(\theta)=h\left(f_{\theta}\right)$.

Define the Markov graph of $\theta \in P_{n}$ to be the directed graph with $n-1$ vertices $\left\{I_{1}, \ldots, I_{n-1}\right\}$ and an edge from $I_{i}$ to $I_{j}$ if $f_{\theta}([i, i+1]) \supset[j, j+1]$. The induced matrix $M(\theta)$ of $\theta \in P_{n}$ is the $(n-1) \times(n-1)$ zero-one adjacency matrix of the Markov graph of $\theta$, so that the $(i, j)$ th entry of $M(\theta)$ is 1 if $f_{\theta}([i, i+1]) \supset[j, j+1]$ and 0 otherwise.

Proposition 2. For $\theta \in P, h(\theta)=\log \rho(M(\theta))$, the spectral radius of the induced matrix of $\theta$.

Propositions 1 and 2 are well known [MS, BGMY].

The entropy of a map is the supremum of the entropies of its orbit types [T, BCov, MN].

If $\theta, \eta \in P$, we say that $\theta$ forces $\eta$, or write $\theta \vdash \eta$, if every map which has an invariant set of type $\theta$ also has an invariant set of type $\eta$.

Proposition 3. $\vdash$ is a partial preorder on $P$, and a partial order on $C$.

That is, $\vdash$ is a reflexive, transitive relation on $P$ and in addition [Ba] is antisymmetric on $C$.

$\theta$ is forcing-maximal in some subset $S$ of $P$ if $\theta \in S$, and for all $\eta \in S$, $\eta \vdash \theta$ implies $\theta \vdash \eta$. It is clear from the definition that if $\theta, \eta \in P$ and $\theta$ forces $\eta$ then $h(\theta) \geq h(\eta)$. Misiurewicz and Nitecki [MN] have determined when one can conclude from $\theta \vdash \eta$ that the inequality is strict.

\section{MISIUREWICZ-NITECKI ORBIT TYPES AND FORCING MAXIMALITY}

For $n$ odd, define $l:=\lfloor(n-1) / 4\rfloor$ so that if $n \equiv 1(\bmod 4)$ then $n=4 l+1$ and if $n \equiv 3(\bmod 4)$ then $n=4 l+3$. Define the orbit type $\theta_{n}$ of period $n$ 
by

$$
j \mapsto \begin{cases}n-2 l-j & \text { if } 1 \leq j<n-2 l \text { and } j \text { odd } \\ j-n+2 l+1 & \text { if } n-2 l \leq j \leq n \text { and } j \text { odd } \\ n-2 l+j-1 & \text { if } 1 \leq j \leq 2 l \text { and } j \text { even } \\ n+2 l-j+2 & \text { if } 2 l<j \leq n \text { and } j \text { even. }\end{cases}
$$

Thus, for example, $\theta_{3}=(123), \theta_{5}=(12453), \theta_{7}=(1473265)$, and $\theta_{9}=$ $(148732695)$. It is easy to see that $\theta_{n}$ is maximodal and that $f_{\theta_{n}}$ has a (local) minimum at $x=1$. One can also verify easily that $\theta_{n}$ is indeed a cycle of period $n$. (See $[\mathrm{MN}]$ for the case $n \equiv 1(\bmod 4)$; the argument is similar for $n \equiv 3(\bmod 4)$.)

For $n \equiv 1(\bmod 4), \theta_{n}$ was defined by Misiurewicz and Nitecki [MN] and played a part in their result characterizing the asymptotic growth rate of the maximal entropy of $n$-cycles. It follows from their results that $\theta_{n}$ (for $n \equiv 1$ $(\bmod 4))$ must at least have near-maximal entropy asymptotically. The main result of this paper is that for $n$ odd, $\theta_{n}$ actually attains maximal entropy in $P_{n}$, the permutations of period $n$, and so in particular in $C_{n}$, the cycles of period $n$.

Theorem 1. For $n$ odd, the cycle $\theta_{n}$ and its dual $\bar{\theta}_{n}$ have maximal entropy among all permutations of period $n$.

Corollary 1. For $n$ odd, $\theta_{n}$ and $\bar{\theta}_{n}$ have maximal entropy among cycles of period $n$.

The proof builds on an idea of Misiurewicz and Nitecki [MN]. We need to show that no other permutation of period $n$ has an induced matrix of larger spectral radius than that of $\theta_{n}$. Using properties of the partial preorder on permutations, it will suffice to focus our attention on a certain class of maximodal permutations. The difficult part of the proof is coming up with a cone in $\mathbf{R}^{n-1}$ which has a particular invariance property and furthermore allows us to demonstrate that the spectral radius of the induced matrix of $\theta_{n}$ dominates that of its competitors.

Since entropy respects the partial preorder on permutations, i.e. if $\theta$ forces $\eta$ then $h(\theta) \geq h(\eta)$, we have that if $\theta_{n}$ does not have maximal entropy among permutations of length $n$, then there is a permutation of length $n$ which is forcing-maximal in $P_{n}$ and has maximal entropy in $P_{n}$ and which satisfies $h(\theta)>h\left(\theta_{n}\right)$.

The following result is in [MN]; an analogue (without the entropy condition) was first proved for cycles in $[\mathrm{J}]$.

Proposition 4. If $\theta \in P_{n}$ is forcing-maximal and has maximal entropy among permutations $P_{n}$, then $\theta$ is maximodal and $f_{\theta}$ has all maximum values above all minimum values, that is,

$$
\begin{aligned}
& \min \left\{\theta(j): j \in[1, n] \text { is a maximum of } f_{\theta}\right\} \\
& \quad<\max \left\{\theta(j): j \in[1, n] \text { is a minimum of } f_{\theta}\right\} .
\end{aligned}
$$

If $M$ is an $m \times m$ matrix, we write $M^{(j)}$ for the $j$ th column of $M, 1 \leq j \leq$ $m$, so $M^{(j)} \in \mathbf{R}^{m}$. For $r=\left(r_{1}, \ldots, r_{m}\right) \in \mathbf{R}^{m}$, we let $|r|:=|r|_{1}=\sum_{i=1}^{m}\left|r_{i}\right|$. 
Proposition 5. If $\theta \in P_{n}$ is a permutation of length $n$, then its induced matrix $M=M(\theta)$ has jth column sum $\left|M^{(j)}\right| \leq \min \{2 j, 2(n-j)\}$.

$\operatorname{Proof}[\mathrm{MN}]$. Let $I_{i}:=[i, i+1] \subset \mathbf{R}, i=1, \ldots, n-1$, and let $f=f_{\theta}$. Then if $i_{1}:=f^{-1}(1)$, we have that $f\left(I_{i}\right) \not \supset I_{1}$ if $i \neq i_{1}, i_{1}-1$, since any interval covering $I_{1}$ must send one of its endpoints to the left of $I_{1}$. Similarly, if $i_{2}:=$ $f^{-1}(2)$ then $f\left(I_{i}\right) \not \supset I_{2}$ for $i \notin\left\{i_{1}, i_{1}-1, i_{2}, i_{2}-1\right\}$. In general, if we let $i_{j}:=$ $f^{-1}(j)$ then $f\left(I_{i}\right) \not \supset I_{j}$ and hence $M_{i j}=0$ for $i \notin\left\{i_{1}, i_{1}-1, \ldots, i_{j}, i_{j}-1\right\}$, and this set has cardinality at most $2 j$. Analogously, but working from the right, we have that $f\left(I_{i}\right) \not \supset I_{j}$ for $i \notin\left\{i_{j+1}, i_{j+1}+1, \ldots, i_{n}, i_{n}+1\right\}$, and the cardinality of this set is at most $2(n-j)$. This establishes Proposition 5.

We say that a maximodal $\theta \in P_{n}, n$ odd, is normalized if $x=1$ is a minimum of $f_{\theta}$ (rather than a maximum). Note that if a maximodal $\theta \in P_{n}$, $n$ odd, is not normalized then its dual $\bar{\theta}$ is. Note also that if $\theta \in P_{n}$ is a permutation of odd length $n, f:=f_{\theta}$ is maximodal with all maximum values above all minimum values, and $\theta$ is normalized, then the $k$ th column $B^{(k)}$ of the induced matrix $B:=M(\theta)$ contains a 0 , where $k=(n-1) / 2$. For otherwise none of the (local) minimum values of $f$ could exceed $k$ (nor could the maximum values be less than $k+1$ ), which is impossible since $f$ has $k+1$ minima, each with distinct image.

Together with Proposition 4 and Proposition 5, this establishes

Proposition 6. Let $n$ be odd and $k=(n-1) / 2$. If $\theta \in P_{n}$ is normalized, forcing maximal, and entropy maximal among permutations of length $n$, with induced matrix $B=M(\theta)$, then the jth column sum $\left|B^{(j)}\right|$ of $B$ satisfies

$$
\left|B^{(j)}\right| \leq \begin{cases}2 j & \text { for } j<k \\ 2 k-1 & \text { for } j=k \\ 2(2 k-j+1) & \text { for } j>k\end{cases}
$$

If $n$ is odd, we will write $A=M\left(\theta_{n}\right)$, suppressing the dependence of $A$ on $n$ in the interest of readability. This should cause no confusion.

Proposition 7. Let $n$ be odd, $k=(n-1) / 2$, and $A=M\left(\theta_{n}\right)$. If $k$ is odd then

$$
A_{j r}=1 \quad \text { iff } \begin{cases}k-j+2 \leq r \leq k+j+1 & \text { for odd } j \leq k-2 ; \\ k-j+1 \leq r \leq k+j & \text { for even } j \leq k-1 ; \\ 2 \leq r \leq 2 k & \text { for } j=k ; \\ j-k-1 \leq r \leq 3 k-j & \text { for odd } j \geq k+2 ;\end{cases}
$$

If $k$ is even then

$$
A_{j r}=1 \quad \text { iff } \begin{cases}k-j+1 \leq r \leq k+j & \text { for odd } j \leq k-1 \\ k-j \leq r \leq k+j-1 & \text { for even } j \leq k-2 \\ 1 \leq r \leq 2 k-1 & \text { for } j=k \\ j-k \leq r \leq 3 k-j+1 & \text { for odd } j \geq k+1 \\ j-k+1 \leq r \leq 3 k-j+2 & \text { for even } j \geq k+2 .\end{cases}
$$

In both cases $A$ is symmetric. 
The proof is a routine verification in the various cases; it is omitted.

In the case when $k$ is odd (i.e., when $n \equiv 3(\bmod 4))$, the matrix $A$ has the following form:

$$
A=\left(\begin{array}{ccccccccccc}
0 & 0 & 0 & \cdots & 0 & 1 & 1 & \cdots & 0 & 0 & 0 \\
0 & 0 & 0 & \cdots & 1 & 1 & 1 & \cdots & 0 & 0 & 0 \\
\vdots & \vdots & \vdots & \ddots & \vdots & \vdots & \vdots & \ddots & \vdots & \vdots & \vdots \\
0 & 0 & 0 & \cdots & 1 & 1 & 1 & \cdots & 0 & 0 & 0 \\
0 & 0 & 0 & \cdots & 1 & 1 & 1 & \cdots & 1 & 1 & 0 \\
0 & 1 & 1 & \cdots & 1 & 1 & 1 & \cdots & 1 & 1 & 0 \\
0 & 1 & 1 & \cdots & 1 & 1 & 1 & \cdots & 1 & 1 & 1 \\
1 & 1 & 1 & \cdots & 1 & 1 & 1 & \cdots & 1 & 1 & 1 \\
1 & 1 & 1 & \cdots & 1 & 1 & 1 & \cdots & 1 & 0 & 0 \\
0 & 0 & 1 & \cdots & 1 & 1 & 1 & \cdots & 1 & 0 & 0 \\
0 & 0 & 1 & \cdots & 1 & 1 & 1 & \cdots & 0 & 0 & 0 \\
0 & 0 & 0 & \cdots & 1 & 1 & 1 & \cdots & 0 & 0 & 0 \\
\vdots & \vdots & \vdots & \ddots & \vdots & \vdots & \vdots & \ddots & \vdots & \vdots & \vdots \\
0 & 0 & 0 & \cdots & 1 & 1 & 1 & \cdots & 0 & 0 & 0 \\
0 & 0 & 0 & \cdots & 1 & 1 & 0 & \cdots & 0 & 0 & 0 \\
0 & 0 & 0 & \cdots & 1 & 1 & 0 & \cdots & 0 & 0 & 0
\end{array}\right) .
$$

Here row and column $k+1$ are those containing all ones.

\section{Corollary 2.}

$$
\left|A^{(j)}\right|= \begin{cases}2 k & \text { for } j<k \\ 2 k-1 & \text { for } j=k \\ 2(2 k-j+1) & \text { for } j>k\end{cases}
$$

We call a vector $t=\left(t_{1}, \ldots, t_{2 k}\right) \in \mathbf{R}^{2 k}$ even if both $\min \left\{i: t_{i} \neq 0\right\}$ and $\min \left\{i: t_{n-i} \neq 0\right\}$ are even. We say $t$ is odd if both of these indices are odd. It is easily checked that $A^{(j)}$ is even for $j \leq k-1$ and $A^{(j)}$ is odd for $j \geq k+1$. $\left(A^{(k)}\right.$ is neither even nor odd.)

Proposition 8. Let $n=2 k+1$ be odd, $A=M\left(\theta_{n}\right)$, and let $B=M(\theta)$, where $\theta \in P_{n}$ is normalized, forcing maximal, and entropy maximal among permutations of length $n$. Then for $j \neq k, 1 \leq j \leq 2 k, B^{(j)}$ has the same parity as $A^{(j)}$. That is, $B^{(j)}$ is even for $j \leq k-1$ and odd for $j \geq k+1$.

Proof. Consider first some $j \leq k-1$. Now let $i_{-}=i_{-}(j)$ be the first point where $f_{\theta}$ does not exceed $j, i_{-}:=\min \left\{i: f_{\theta}(i) \leq j\right\} \in\{1, \ldots, n\}$. Then $i_{-}$must be a minimum for $f_{\theta}$, since otherwise by maximodality it would be a maximum, hence different from 1 by normalization, and so we would have $f_{\theta}\left(i_{-}-1\right)<f_{\theta}\left(i_{-}\right) \leq j$, contradicting the definition of $i_{-}$. But then maximodality tells us that $i_{-}$is odd. A similar argument shows that $i_{+}:=$ $\max \left\{i: f_{\theta}(i) \leq j\right\}$ must also be a minimum and hence also odd. But this implies that $B^{(j)}$ is even, since if $B^{(j)}=\left(t_{1}, \ldots, t_{2 k}\right)$ then $\min \left\{i: t_{i} \neq 0\right\}=i_{-}-1$ and $\min \left\{i: t_{n-i} \neq 0\right\}=n-i_{+}$, both of which are even.

An analogous argument shows that $i^{-}:=\min \left\{i: f_{\theta}(i) \geq j+1\right\}$ and $i^{+}:=$ $\max \left\{i: f_{\theta}(i) \geq j+1\right\}$ are maxima and hence $B^{(j)}$ is odd for $j \geq k+1$. 


\section{INVARIANCE OF THE CONE $\mathscr{P}$ UNDER $A^{2}$}

Let $n$ be odd, $n=2 k+1$. Consider again the Euclidean space $\mathbf{R}^{n-1}=\mathbf{R}^{2 k}$. A vector $r$ in this space will now be denoted as

$$
r=(u, v)=\left(u_{1}, \ldots, u_{k}, v_{k}, \ldots, v_{1}\right) .
$$

This notation is different from the one used in the previous section; it turns out to be more convenient to describe the following class of "peaked" vectors.

Definition 2. We say that $r$ belongs to the class of vectors $\mathscr{P} \subset \mathbf{R}_{+}^{2 k}=\{x \in$ $\left.\mathbf{R}^{2 k}: x \geq 0\right\}$ if the following two conditions hold:

(1.e) $v_{k} \geq u_{k} \geq u_{k-1} \geq v_{k-1} \geq v_{k-2} \geq \cdots \geq u_{2} \geq u_{1} \geq v_{1}$, if $k$ is even (i.e., if $n \equiv 1(\bmod 4))$.

(1.o) $v_{k} \geq u_{k} \geq u_{k-1} \geq v_{k-1} \geq v_{k-2} \geq \cdots \geq v_{2} \geq v_{1} \geq u_{1}$, if $k$ is odd (i.e., if $n \equiv 3(\bmod 4))$.

(2) $v_{k}-u_{k} \geq u_{k-1}-v_{k-1} \geq v_{k-2}-u_{k-2} \geq \cdots \geq(-1)^{k}\left(u_{1}-v_{1}\right) \geq 0$, or, separating in cases:

(2.e) $v_{k}-u_{k} \geq u_{k-1}-v_{k-1} \geq v_{k-2}-u_{k-2} \geq \cdots \geq v_{2}-u_{2} \geq u_{1}-v_{1} \geq 0$, if $k$ is even $(n \equiv 1(\bmod 4))$;

(2.o) $v_{k}-u_{k} \geq u_{k-1}-v_{k-1} \geq v_{k-2}-u_{k-2} \geq \cdots \geq u_{2}-v_{2} \geq v_{1}-u_{1} \geq 0$, if $k$ is odd $(n \equiv 3(\bmod 4))$.

Note that here, as in the definition of $A$, we suppress the dependence of $\mathscr{P}$ on $n$.

Remark. Condition (2) implies the following "half" of (1):

$$
v_{k} \geq u_{k}, \quad u_{k-1} \geq v_{k-1}, \quad v_{k-2} \geq u_{k-2}, \ldots
$$

Therefore, we need only assume in (1) the remaining half:

$$
u_{k} \geq u_{k-1}, \quad v_{k-1} \geq v_{k-2}, \ldots
$$

Consequently, if we assume condition (2) then condition (1) is equivalent to the following:

$$
\min \left(v_{j+1}, u_{j+1}\right) \geq \max \left(v_{j}, u_{j}\right) \text { for all } j=1,2, \ldots, k-1 .
$$

In what follows we shall assume that $k$ is odd (i.e., $n \equiv 3(\bmod 4))$. The arguments for the case when $k$ is even are analogous.

Consider the matrix $A=M\left(\theta_{n}\right)$ of the orbit type $\theta_{n}$ (see Proposition 7). We now prove the following result.

Proposition 9. The cone $\mathscr{P}$ is invariant under $A^{2}$, i.e., $\mathscr{P} A^{2} \subset \mathscr{P}$.

Proof. Take a vector $(w, z)$ in $\mathscr{P}$, and denote $(u, v)=(w, z) A,(x, y)=$ $(u, v) A$. We prove that $(x, y) \in \mathscr{P}$. Note that the vector $(u, v)$ in general does not belong to $\mathscr{P} ; \mathscr{P}$ is not invariant under $A$. Let us define, for $p=$ $1,2, \ldots, k$,

$$
U(p)=\sum_{j=p}^{k} u_{j} \quad \text { and } \quad V(p)=\sum_{j=p}^{k} v_{j}
$$

We define also

$$
U(k+1)=0 ; \quad V(0)=V(1)
$$


and we note that $U$ and $V$ are (weakly) decreasing in $p$. The numbers $W(p)$ and $Z(p)$ are defined similarly, in terms of the $w_{j}$ and $z_{j}$ respectively. Equality $(x, y)=(u, v) A$ then becomes

$$
\begin{gathered}
x_{s}=U(k+2-s)+V(k-s), \quad \text { where } s \text { is odd }, s=1,3, \ldots, k ; \\
x_{s+1}=U(k-s)+V(k-s), \quad s \text { odd }, s=1,3, \ldots, k ; \\
y_{s}=U(k+1-s)+V(k+1-s), \quad s \text { odd }, s=1,3, \ldots, k ; \\
y_{s+1}=U(k-1-s)+V(k+1-s), \quad s \text { odd }, s=1,3, \ldots, k .
\end{gathered}
$$

We have analogous formulas for $(u, v)=(w, z) A$.

Step 1. Proof of condition (1). By our remark above, we need only prove that $x_{k+1-s} \geq x_{k-s}$ and $y_{k-s} \geq y_{k-1-s}$ for $s$ odd, $s=1,3, \ldots, k-2$. For such $s$, we have

$$
x_{k+1-s}-x_{k-s}=V(s-1)-V(s+1),
$$

which is nonnegative since $V$ is decreasing. The second inequality is also true:

$$
y_{k-s}-y_{k-1-s}=U(s)-U(s+2) \geq 0
$$

for all $s$ odd, $s=1,3, \ldots, k-2$.

We now proceed to prove (2.o).

Step 2. We first prove the inequalities

$$
y_{k+1-s}-x_{k+1-s} \geq x_{k-s}-y_{k-s},
$$

or

$$
y_{k-s}+y_{k+1-s}-x_{k-s}-x_{k+1-s} \geq 0
$$

for $s$ odd, $s=1,3, \ldots, k-2$. We have

$$
\begin{aligned}
y_{k-s}+ & y_{k+1-s}-x_{k-s}-x_{k+1-s} \\
= & U(s)+V(s+2)+U(s)+V(s) \\
& -[U(s+1)+V(s+1)+U(s+1)+V(s-1)] \\
= & 2 u_{s}-v_{s-1}-v_{s+1},
\end{aligned}
$$

for $s$ odd, $s=3,5, \ldots, k-2$. Recall now that $(u, v)=(w, z) A$. Reasoning as above, using $W(p)$ and $Z(p)$ instead of $U(p)$ and $V(p)$ respectively, we obtain

$$
\begin{aligned}
2 u_{s}-v_{s-1}-v_{s+1}= & {\left[z_{k-s}+z_{k+1-s}-w_{k-s}-w_{k+1-s}\right] } \\
& +\left[z_{k+2-s}-w_{k+1-s}\right]+\left[z_{k-s}-w_{k-1-s}\right],
\end{aligned}
$$

for $s$ odd, $s=3,5, \ldots, k-2$. The first expression in brackets is nonnegative by assumption $(2)$ on the vector $(w, z)$. The last two are also nonnegative by condition (1.0) on $(w, z)$.

For the special case $s=1$ we obtain

$$
\begin{aligned}
y_{k-1} & +y_{k}-x_{k-1}-x_{k}=2 u_{1}-v_{2} \\
& =\left[z_{k}+z_{k-1}-w_{k}-w_{k-1}\right]+\left[z_{k-1}-w_{k-2}\right] \geq 0,
\end{aligned}
$$

since, again by assumption (2), the expression in the first bracket in nonnegative, and so is that in the second bracket by condition (1.0).

Step 3. The proof of the remainder of $(2.0)$ for $(x, y)$, that

$$
x_{k-s}-y_{k-s} \geq y_{k-s-1}-x_{k-s-1}
$$


for $s$ odd, $s=1,3, \ldots, k-2$, is very similar to the previous step and is therefore omitted.

Step 4. Finally, we must show that $y_{1}-x_{1} \geq 0$. A direct computation yields

$$
y_{1}-x_{1}=u_{k}-v_{k-1}=z_{2}+\left(z_{1}-w_{1}\right) \geq 0,
$$

since $z_{1} \geq w_{1}$.

This establishes Proposition 9.

\section{ENTROPY MAXIMALITY}

We introduce some convenient notation. Given a positive integer $m$ (which for us will just be $m=2 k=n-1)$ and $1 \leq j \leq j^{\prime} \leq m$, we write $\left\langle j, j^{\prime}\right\rangle:=$ $\sum_{i=j}^{j^{\prime}} e^{i}=(0, \ldots, 0,1, \ldots, 1,0, \ldots, 0) \in \mathbf{R}^{m}$, where $e^{i}$ is the $i$ th standard basis vector in $\mathbf{R}^{m}$. For consistency we write $\langle j\rangle$ for $e^{j}=\langle j, j\rangle \in \mathbf{R}^{m}$. We say $\left\langle j, j^{\prime}\right\rangle$ is centered if $j^{\prime}=m+1-j$.

Proposition 10. Let $n=2 k+1$ be odd, $k>1$, and $A=M\left(\theta_{n}\right)$. Let $B=M(\theta)$, where $\theta \in P_{n}$ is normalized, forcing-maximal, and has maximal entropy among permutations of length $n$. If $r \in \mathscr{P}$, then $r A \geq r B$.

Proof. We consider the case $n \equiv 3(\bmod 4)$, i.e. $k=(n-1) / 2$ odd; the case $n \equiv 1(\bmod 4)$ is handled similarly. If $A$ and $B$ are as above and $r \in \mathscr{P}$, we want to show that $(r A)_{j} \geq(r B)_{j}$, in other words $r A^{(j)} \geq r B^{(j)}$, for $j=1, \ldots, 2 k$.

Note first that from Proposition 6 and the corollary to Proposition 7, we have $\left|B^{(j)}\right| \leq\left|A^{(j)}\right|$. Thus $B$ has no more ones in the $j$ th column than $A$ does.

It is easiest to see what we want to show when $j$ is even. For in this case, $A^{(j)}=\langle k-j+1, k+j\rangle$ if $j \leq k-1$ and $A^{(j)}=\langle j-k, 3 k-j+1\rangle$ if $j \geq k+1$, both of which are centered, and $r$ is "peaked towards the center" (by (1.o) in the definition of $\mathscr{P})$. That is to say, if $r=\left(u_{1}, \ldots, u_{k}, v_{k}, \ldots, v_{1}\right) \in \mathscr{P} \subset \mathbf{R}_{+}^{2 k}$, then $r A^{(j)}=\sum_{i=k-j+1}^{k}\left(u_{i}+v_{i}\right)$ if $j \leq k-1$ (and $r A^{(j)}=\sum_{i=j-k}^{k}\left(u_{i}+v_{i}\right)$ if $j \geq k+1$ ) and these are the $2 j$ (or $2(2 k-j+1)$ ) largest coordinates of $r$ (by (1.o) in the definition of $\mathscr{P})$. Thus $r A^{(j)} \geq r B^{(j)}$ for $j$ even.

Now let $j$ be odd, and assume $j \leq k-2$. Then $A^{(j)}=\langle k-j+2, k+1+j\rangle$, which unfortunately is not centered. Assume, for a contradiction, that $r B^{(j)}>$ $r A^{(j)}$.

Claim: $\langle k-j, k+j+1\rangle B^{(j)}=2 j$. (Note that $\langle k-j, k+j+1\rangle A^{(j)}=$ $\langle 1,2 k\rangle A^{(j)}=\left|A^{(j)}\right|=2 j$.) Otherwise, we would have $\langle k-j, k+j+1\rangle B^{(j)} \leq$ $2 j-1$. Now from the definition of $\mathscr{P}$, we know that the $2 j+2$ largest components of $r$ are the $2 j+2$ central ones, that is, $r_{k-j}$ to $r_{k+j+1}$. The idea here is that at least three of these are "missed" by $B^{(j)}$, but only the $2 j$ th and $(2 j+1)$ th largest are missed by $A^{(j)}$. More precisely, referring to part (1.o) of the definition of $\mathscr{P}$ we can write

$$
\begin{aligned}
r^{(1)} & =r_{k+1} \geq r^{(2)}=r_{k} \geq r^{(3)}=r_{k-1} \\
& \geq \cdots \geq r^{(2 k-2)}=r_{2 k-1} \geq r^{(2 k-1)}=r_{2 k} \geq r^{(2 k)}=r_{1} .
\end{aligned}
$$

Then $r A^{(j)}-r B^{(j)}=r\left(A^{(j)}-B^{(j)}\right) \geq r^{(2 j+2)}-r^{\left(i_{0}\right)}$, where $i_{0} \geq 2 j+3$, so the last difference is nonnegative. This establishes the claim. 
Thus we know that $B^{(j)}=\langle k-j, k+j+1\rangle-\left\langle i_{1}\right\rangle-\left\langle i_{2}\right\rangle$ for some $i_{1}, i_{2}$ satisfying $k-j \leq i_{1}, i_{2} \leq k+j+1$, so that $B_{i_{1}}^{(j)}=B_{i_{2}}^{(j)}=0$. Then

$$
\begin{aligned}
0 & <r B^{(j)}-r A^{(j)}=r_{k-j}+r_{k-j+1}-r_{i_{1}}-r_{i_{2}} \\
& =r^{(2 j+1)}+r^{(2 j)}-r^{\left(i_{1}^{\prime}\right)}-r^{\left(i_{2}^{\prime}\right)},
\end{aligned}
$$

where $1 \leq i_{1}^{\prime}<i_{2}^{\prime} \leq 2 j+2$. For this positivity claim to hold, we must have $i_{2}^{\prime}=2 j+2$, so $i_{2}=k+j+1$. Now $B^{(j)}$ is even by Proposition 8 , so since $n-\left(i_{2}-1\right)=k-j+1$ is odd, we must have that $i_{1}=k+j$. Then the positivity claim boils down to

$$
0<r B^{(j)}-r A^{(j)}=-\left(r_{k+j}-r_{k-j+1}\right),
$$

but this contradicts part (2) of the definition of $\mathscr{P}$. This establishes the result for $j$ odd, $j \leq k-2$.

For $j$ odd, $j \geq k+2$ we have a similar argument. For $j=k$, we have $r A^{(k)}-r B^{(k)} \geq r_{i_{3}}-r_{1} \geq 0,1 \leq i_{3} \leq 2 k$. Analogous arguments holds for $k$ even.

We now are in a position to finish off the proof of the main theorem.

Proof of Theorem 1. Let $w:=(1, \ldots, 1) \in \mathbf{R}_{+}^{2 k}, k=(n-1) / 2$. Then $w \in \mathscr{P}$. Let $A=M\left(\theta_{n}\right)$. Note that $w A$ satisfies $(w A)_{j}=\left|A^{(j)}\right|$, so by the corollary to Proposition 7 we have

$$
(w A)_{j}= \begin{cases}2 j & \text { for } j<k \\ 2 k-1 & \text { for } j=k \\ 2(2 k-j+1) & \text { for } j>k\end{cases}
$$

and thus $w A \in \mathscr{P}$. Therefore, since $\mathscr{P} A^{2} \subset \mathscr{P}$, it follows that $w A^{m} \in \mathscr{P}$ for all $m \geq 1$.

Let $\theta \in P_{n}$ be entropy maximal and forcing maximal among all permutations of length $n$, and let $\theta$ be normalized. We will show that $h\left(\theta_{n}\right) \geq h(\theta)$. Let $B=M(\theta)$.

Claim: $w A^{m} \geq w B^{m}$ for all $m \geq 1$. Since $w \in \mathscr{P}$, Proposition 10 implies the claim for the case $m=1$. To prove the general case, we use induction on $m$. Assume $w A^{m} \geq w B^{m}$. Then, applying Proposition 10 and the fact that $w A^{m} \in \mathscr{P}$, we get that

$$
w A^{m+1}=\left(w A^{m}\right) A \geq\left(w A^{m}\right) B \geq\left(w B^{m}\right) B=w B^{m+1},
$$

where for the last inequality we used the induction hypothesis and the fact that $B$ is nonnegative. This establishes the claim.

If $M$ is a nonnegative $2 k \times 2 k$ matrix we can write $\|M\|:=\sum_{i, j} M_{i j}=$ $|w M|$. The spectral radius $\rho(M)$ satisfies $\rho(M)=\lim _{m \rightarrow \infty}\left\|M^{m}\right\|^{1 / m}$. We have

$$
\begin{aligned}
h\left(\theta_{n}\right) & =\log \rho(A)=\log \lim _{m \rightarrow \infty}\left\|A^{m}\right\|^{1 / m}=\log \lim _{m \rightarrow \infty}\left|w A^{m}\right|^{1 / m} \\
& \geq \log \lim _{m \rightarrow \infty}\left|w B^{m}\right|^{1 / m}=\log \lim _{m \rightarrow \infty}\left\|B^{m}\right\|^{1 / m}=\log \rho(B)=h(\theta) .
\end{aligned}
$$

So $\theta_{n}$ has maximal entropy and we are done. 


\section{REFERENCES}

[AKM] R. L. Adler, A. G. Konheim, and M. H. McAndrew, Topological entropy, Trans. Amer. Math. Soc. 114 (1965), 309-319.

[Ba] S. Baldwin, Generalizations of a theorem of Sarkovskii on orbits of continuous real-valued functions, Discrete Math. 67 (1987) 111-127.

[Be] C. Bernhardt, The ordering on permutations induced by continuous maps of the real line, Ergodic Theory Dynamical Systems 7 (1987), 155-160.

[BCop] L. Block and W. A. Coppel, Stratification of continuous maps of an interval, Trans. Amer. Math. Soc. 297 (1986), 587-604.

[BCov] L. Block and E. M. Coven, Approximating entropy of maps of the interval, Banach Center Publ. 22.

[BGMY] L. Block, J. Guckenheimer, M. Misiurewicz, and L. S. Young, Periodic points and topological entropy of one-dimensional maps, Lecture Notes in Math., vol. 819, Springer-Verlag, Berlin and New York, 1980, pp. 18-34.

[BH] L. Block and D. Hart, Orbit types for maps of the interval, Ergodic Theory Dynamical Systems 7 (1987), 161-164.

[Bow] R. Bowen, Entropy for group endomorphisms and homogeneous spaces, Trans. Amer. Math. Soc. 153 (1971), 401-414.

[BowF] R. Bowen and J. Franks, The periodic points of maps of the disk and the interval, Topology 15 (1976), 337-342.

[J] I. Jungreis, Some results on the Sarkovskii partial ordering of permutations, preprint, Harvard Univ., 1987.

[MN] M. Misiurewicz and Z. Nitecki, Combinatorial patterns for maps of the interval, preprint.

[MS] M. Misiurewicz and W. Szlenk, Entropy of piecewise monotone mappings, Studia Math. 67 (1980), 45-63

[N] Z. Nitecki, Topological dynamics on the interval, Ergodic Theory and Dynamical Systems II (Proc. Special Year, Maryland 1979-80, Ed., A. Katok), Birkhäuser, Basel, Boston, Mass., and Stuttgart, 1982, pp. 1-73.

[T] Y. Takahashi, A formula for topological entropy of one-dimensional dynamics, Sci. Papers College Gen. Ed. Univ. Tokyo 30 (1980), 11-22.

Department of Mathematics, University of California, Berkeley, California 94720

Current address, William Geller, Department of Mathematics, University of Maryland, College Park, Maryland 20742

E-mail address: geller@math.umd.edu

Current address, Juan Tolosa: Department of Mathematics, Stockton State College, Pomona, New Jersey 08420

E-mail address: tolosa@polot.njin.net 